\title{
Sextortion: una minaccia (anche) per la nostra lingua?
}

\section{Sara Giovine}

PUBBLICATO: 30 SETTEMBRE 2019

$\mathbf{L}$

a parola sextortion (attestata anche nella variante sextorsion, graficamente scorretta) indica la pratica di chi estorce denaro, favori sessuali o altro ai danni di una persona, con la minaccia di rendere pubblici contenuti compromettenti di natura sessuale (messaggi di testo, foto o video). Dal punto di vista linguistico, la voce rappresenta un prestito integrale dall'inglese sextortion, una parola macedonia (secondo la denominazione coniata da Bruno Migliorini per indicare i composti formati dalla fusione di due o più parole; per cui si vedano anche le schede relative a Brexit e apericena), formata dalla fusione dei sostantivi sex 'sesso' e (ex)tortion 'estorsione'. È plausibile che la nostra voce sia stata formata sul modello di di sexting 'invio, tramite le nuove tecnologie telematiche, di materiale sessualmente esplicito', un'altra parola macedonia, crasi di sex 'sesso' e (tex)ting, gerundio di to text 'inviare messaggi', attestata in inglese dal 2005 (come indicato nell'Oxford English Dictionary).

Secondo quanto indicato dai dizionari e altri strumenti lessicografici inglesi (tra cui il Cambridge Dictionary e il portale Lexico.com), le prime attestazioni di sextortion in inglese risalirebbero agli anni '50 del Novecento, e più precisamente al 5 aprile del 1950, quando il termine viene impiegato in un articolo del "Los Angeles Times" per indicare le intimidazioni mosse da due giovani donne ai danni di un testimone federale, per indurlo a modificare la sua deposizione nel corso di un processo, con la minaccia di diffondere informazioni e immagini personali di natura sessuale:

The sensational "sextortion" phase of the Bridges trial still remains to be settled and will be aired in
Judge Louis E. Goodman's court on April Io. Two women, attractive brunet Mrs Green Teixera, 29, and
Mrs Jean Simons Murray, 33, a strawberry blonde, face trial on charges of conspiring to intimidate a
government witness. ['La sensazionale fase "sextortion" del processo Bridges resta ancora aperta e sarà
affrontata pubblicamente nella corte del giudice Louis E. Goodman il Io aprile. Due donne, l'attraente,
bruna Green Teixera, di 29 anni, e Jean Simons Murray, di 33, biondo ramata, sono sotto processo con
l'accusa di aver cospirato per intimidire un testimone federale']. (Sextortion Charges To Come Up Next
Week, "Los Angeles Times", April 5, 1950, p. ro)

A partire da tale significato specifico, di sfruttamento della storia sessuale di un testimone per spingerlo a mutare la sua testimonianza, si sarebbe in seguito diffuso e affermato un uso del termine con un significato più ampio e generico, a indicare qualsiasi forma di estorsione a sfondo sessuale che induca la vittima a compiere un'azione contro la propria volontà. In particolare, nel corso dei successivi decenni la voce viene impiegata in inglese specialmente per designare le due differenti e più comuni tipologie di estorsione sessuale: l'estorsione di favori sessuali da parte di chi riveste una posizione di autorità tramite l'intimidazione e l'abuso di potere, talora in cambio di favori e concessioni di vario tipo; e lo sfruttamento di informazioni o contenuti personali compromettenti (messaggi di testo, foto o video), con la minaccia di renderli pubblici, per forzare la vittima a concedere denaro, favori sessuali, ulteriore materiale di contenuto pornografico, o altro ancora.

Nel nuovo millennio, con l'avvento delle nuove tecnologie e la nascita dei social network, lo scambio di contenuti digitali, inclusi quelli di natura sessuale, diviene più semplice e immediato e ciò comporta un inevitabile incremento della seconda tipologia di estorsione: nella sua più comune modalità di attuazione, la vittima viene adescata su Facebook o altri social network da una persona che si serve di 
un profilo falso con immagini di donne o uomini avvenenti; una volta ottenuta la fiducia della vittima, l'adescatore la coinvolge in attività sessuali online, registrandole a sua insaputa, o la convince all'invio di foto o video compromettenti, per i quali viene infine ricattata, nella maggior parte dei casi con la richiesta di denaro. Negli Stati Uniti la crescita esponenziale del fenomeno fa si che nel 2009 venga addirittura creato uno specifico portale internet (www.sextortion.org) per la prevenzione e l'acquisizione di una maggiore consapevolezza da parte dei cittadini nell'utilizzo delle tecnologie digitali, mentre il moltiplicarsi di articoli e pubblicazioni specificatamente dedicate all'argomento contribuisce alla sempre maggiore diffusione della parola, che nel secondo decennio degli anni Duemila comincia a essere usata, come prestito dall'inglese, anche in diversi paesi d'Europa.

In italiano la prima attestazione del termine, con specifico riferimento alle estorsioni sessuali proprie dell'era digitale, risale al 20I2, quando compare, tra virgolette, in un articolo del "Corriere della Sera" che dà notizia della condanna di un hacker messicano, arrestato l'anno precedente per aver ricattato più di 230 persone, dopo averne violato i dispositivi informatici e sottratto foto e informazioni di natura strettamente personale:

Spiava le sue vittime attraverso le webcam, entrava negli hard disk, rubava foto e informazioni. E poi le ricattava. Torna a far discutere l'incredibile vicenda dell'hacker messicano Luis Mijangos, arrestato nel settembre del 20II, con l'accusa di aver spiato più di 230 persone, alcune delle quali minorenni. [...] L'accusa è di estorsione e "sextortion", la condanna sette anni di carcere. (Marta Serafini, L'hacker che rubava le vite via webcam, Corriere.it, sez. Esteri, I5/3/2012)

Una prima occorrenza della parola, nella variante impropria sextorsion (con parziale adattamento della grafia alla pronuncia e probabile influsso dell'italiano estorsione) era in realtà già apparsa nella stampa italiana nel 1950, in un breve articolo di cronaca che riportava la notizia del ricatto perpetrato da due ragazze ai danni di un professore universitario americano, i cui incontri intimi con una delle due giovani erano stati registrati per mezzo di un apparecchio nascosto (l'attestazione è di pochi mesi precedente alla prima occorrenza inglese, ma è plausibile ritenere che il termine circolasse in inglese prima che nella nostra lingua). Si tratta tuttavia di una prima attestazione che resta del tutto isolata per più di mezzo secolo, oltre a risultare probabilmente motivata dalla decisione del giornalista di riportare la forma originaria impiegata nelle fonti della notizia.

Una nuova forma di ricatto è stata adottata ad Hollywood e si chiama "sextorsion". Protagonisti: Mister B. Klekner, professore universitario, e due graziose fanciulle poco più che ventenni, Helen Keller e T. Hugues. Il vecchio professore incontrò Helen in ascensore e si innamorò di lei, prese in affitto un appartamento e vi installò la fanciulla. [...] Allora per obbligare il vecchio avaro a sciogliere i cordoni alla borsa, Helen di accordo con la sua amica installò nell'appartamentino dove avevano luogo i convegni intimi un apparecchio registratore, poi fece sentire i dischi al povero professore, il quale per tre anni fu così ricattato e sborsò novemila dollari. (B. Cerdonio, Registratore d'amore, "Stampa Sera", sez. Attualità e aneddoti, p. 3, 3/r/1950)

Bisogna quindi attendere gli anni dieci del nuovo secolo, con la diffusione anche in Italia delle nuove estorsioni a sfondo sessuale veicolate dalla rete, per assistere all'effettiva introduzione del termine nella nostra lingua e alla sua progressiva circolazione nell'uso, che aumenta di pari passo alla crescita esponenziale del fenomeno nel Paese: stando infatti ai dati resi noti dalla Polizia postale, che nel 2015 ha creato un gruppo investigativo apposito per combattere il nuovo crimine informatico, tra il $2013 \mathrm{e}$ il 2018 sarebbero stati oltre cinquemila i casi di "sextortion" in Italia, con un aumento delle denunce del 500\% negli ultimi tre anni. Tali dati, a partire dal 2015, e con una frequenza che diviene più consistente tra il 2017 e il 2018 , vengono riportati dalle principali testate giornalistiche, e soprattutto da blog e periodici online che affrontano l'argomento per sensibilizzare gli utenti sui pericoli del web 
e spiegare in che cosa consistano e come affrontare simili estorsioni:

Il Sextortion, o ricatto sessuale, consiste nel minacciare di rendere pubbliche le informazioni private di una vittima a meno che questa non paghi dei soldi all'estorsore. In un'era digitale come la nostra, le informazioni potrebbero includere frammenti di messaggi di testo sessuali (sexts), foto private e anche video. (Come funziona la Sextortion e perché è meglio denunciare, Agi.it, sez. Cronaca, 24/3/2018)

È la cosiddetta sextortion [...]. Lo schema della truffa è quello classico: la vittima incontra online una persona attraente e disponibile che propone una sessione intima reciproca in video con Skype o simili. La persona si rivela poi essere un ricattatore che registra l'esibizione della vittima e chiede denaro per non diffondere la registrazione agli amici della vittima stessa. (Come si bloccano $i$ video di pornoricatto (sextortion) su Youtube, attivissimo.blogspot.com, 19/10/2018)

Ciò contribuisce alla discreta affermazione nell'uso della voce, di cui si rinvengono circa 42.600 occorrenze nelle pagine italiane di Google (con una ricerca condotta il i2/8/2019), sebbene la sua circolazione risulti per il momento circoscritta al web e a qualche pubblicazione specialistica di ambito psicologico e/o criminologico: il termine, anche in conseguenza della sua recente introduzione in italiano, non è ancora registrato da alcun dizionario o repertorio lessicografico, oltre a comparire in appena una decina di casi negli archivi dei maggiori quotidiani nazionali (2o in quelli della "Stampa", I3 in quelli di "Repubblica" e meno di io in quelli del "Corriere della Sera"). Prevedibilmente minori i numeri relativi alla frequenza d'uso della variante sextorsion, di cui si registrano comunque 4.330 risultati nelle pagine italiane di Google ma appena un paio di attestazioni nei quotidiani, sempre più attenti alla corretta trascrizione grafica del prestito inglese. Minoritarie anche le occorrenze del termine nelle varianti con mancata fusione dei due elementi componenti, che vengono semplicemente accostati: in rete si registrano infatti (il I9/8) 4.350 esempi di sexestorsion, I.380 di sexestortion, 4I3 di sexextortion e appena 9 di sexextorsion.

Maggiore incertezza si registra invece nell'attribuzione del genere al sostantivo, come spesso avviene per gli anglismi di recente introduzione nella nostra lingua (per cui si veda la scheda di Raffaella Setti), con vistose oscillazioni nell'uso, talora anche all'interno dello stesso articolo, tra il femminile (dovuto all'analogia con il genere del traducente italiano "estorsione", formalmente affine all'inglese), e il maschile (per analogia invece con "ricatto" e forse anche con l'iperonimo "reato", o per semplice default, dato che di norma i prestiti inglesi divengono in italiano di genere maschile), per quanto quest'ultimo, da una ricerca in rete, risulti al momento largamente maggioritario (r.o6o occorrenze della stringa di ricerca "la sextortion" contro le 2.740 di "il sextortion").

Infine, come possibili traducenti di sextortion, possiamo forse suggerire il ricorso a espressioni quali estorsione sessuale in rete o ricatto sessuale in rete, formate da sostantivi già esistenti in italiano, accompagnati dall'aggettivo sessuale e dal complemento in rete che qualifichino l'ambito di afferenza del reato: tali sintagmi potrebbero tuttavia non risultare altrettanto efficaci della voce inglese, più sintetica e immediata. Un'alternativa più convincente può essere rappresentata dalla neoformazione pornoricatto, che si rivela già discretamente attestata nell'uso, spesso impiegata come traducente dagli stessi articoli che trattano l'argomento (in rete si rinvengono infatti 23.900 occorrenze della variante analitica porno-ricatto, anche senza trattino, e 3.630 di quella sintetica, univerbata, pornoricatto). La forma avrebbe poi il vantaggio di richiamare per analogia non solo altri composti formati con il prefissoide porno-, da tempo attestati nella nostra lingua (come pornodivo, pornorivista e pornocassetta), ma anche la neoformazione semanticamente affine pornovendetta, di recente proposta dal gruppo Incipit come possibile traducente del sintagma inglese revenge porn e già largamente diffusa nell'uso corrente. 


\section{Cita come:}

Sara Giovine, Sextortion: una minaccia (anche) per la nostra lingua?, "Italiano digitale", 2019, X, 2019/3 (luglio-settembre)

DOI: $10.35948 / 2532-9006 / 2020.3210$

Copyright 2019 Accademia della Crusca

Pubblicato con licenza creative commons CC BY-NC-ND 